\title{
Reprodutibilidade de uma escala odontológica proposta como indicador de saúde bucal em crianças e adolescentes HIV ${ }_{+} /$SI DA
}

\author{
Reproducibility of a scale for oral health \\ among children and adolescents HIV /AIDS
}

Juliana Alvares Duarte Bonini Campos ${ }^{1}$

Leonor de Castro M onteiro Loffredo ${ }^{1}$

${ }^{1}$ Departamento de Odontologia Social, Faculdade de Odontologia deAraraquara,

Universidade Estadual

Paulistajúlio de M esquita

Filho. Rua Humaitá 1.680,

Centro. 14801-385

Araraquara SP.

jucampos@foar.unesp.br
Abstract The aim of this study was to investigate the intraobserver reproducibility of a Portuguese version scale used to evaluate aspects of oral health among children and adolescents H IV +. The scale was proposed by Balbo and a questionnaire was applied in two occasions, within a one week period, to 27 children and adolescents. The reproducibility was estimated by kappa statistics by point (k). With relation to the mother, it was reached a maximum of agreement for items related to the importance of maintaining the oral health, diary tooth brushing and the individual utilization of dental brush; breath and esthetics presented a good agreement. Regarding the children, a regular agreement was observed on the dental flossuse. In contrast, the respondents had difficulties in reporting the name and address of the professionals that gave guidance in prevention activities to them. The k-values for perception, care and promotion were $0.48,0.21$ and 0.64 respectively, pointing to reproducibility levels classified as regular, fair and good. Given the different levels of reproducibility offered by the dental scale for HIV + oral health, we suggest that it could be modified, especially in the domain of care. Key words Reproducibility, HIV/AIDS, Oral health, $\mathrm{H}$ ealth services
Resumo 0 objetivo deste trabalho foi estimar a reprodutibilidadedeuma escala odontológica para avaliação da saúde bucal de crianças e adolescentes HIV $_{+}$. A amostra, com delineamento não probabilístico, constituiu-se de 27 crianças e adolescentes HIV . A escala foi aplicada, em duas ocasiões, com intervalo de sete dias. Estimou-se a reprodutibilidade intraexaminador pela estatística Kappa. Nas questões relativas à mãe, houve concordância máxima nos itens referentes à importância da saúde bucal, hábito de escovação diária e utilização de escova dental. A procura de atendimento odontológico motivado por estética epara manutenção de hálito puro apresentou concordância boa. $N$ as questões relativas à criança, observou-se concordância regular nos itens referentes ao fio dental. Com relação à organização do sistema de saúde, chama atenção a dificuldadedos respondentes em relatar o local e o profissional que realizou orientações educativas preventivas. A reproduti bilidade dos domínios percepção, cuidado e promoção foi de 0,48, 0,21 e 0,64, respectivamente. Sugere se a necessidade de reestruturação das questões componentes dos al gor itmos propostos pela escala para as dimensões de percepção ecuidado, a par da necessidadeda saúde bucal ser inserida em programas de atendimento a portadores HIV

Palavras-chave Reprodutibilidade, HIV/SIDA, Saúde bucal, Serviços de saúde 
Introdução

As doenças bucais de alta prevalência ou de alta letal idade representam importante problema de saúde pública, pois causam impacto nos portadores, exercendo efeito negativo na qualidade de vida dos mesmos.

Dentre estas, destacam-se os pacientes com diagnóstico positivo para HIV (HIV $)$ que, devido à infecção, possuem uma diminuição progressiva em número e atividade dos linfócitos $T$ CD $4_{{ }^{\prime}}$ comprometen do a imunidade celular, deixando o hospedeiro suscetível ao desenvolvimento de infecções oportunistas e neoplasmas ${ }^{1,2}$. A cavidade bucal é particularmente suscetível à infecção, sendo uma fonte importante frequentementeutilizada para estimar o diagnóstico e prognóstico em pacientes $\mathrm{HIV}_{+}{ }^{3}$.

Chapple e $\mathrm{Hamburger}^{3}$ revisaram as principais lesões bucais queacometem estes pacientese destacam a leucoplasia, Sarcoma de Kaposi, linfoma de H odgkin, candidose bucal, herpes simples, úlceras bucais, doença periodontal, doenças das glândulas sal ivares elesões bucais induzidas por medicamentos.

Para Coulter et al. ${ }^{4}, 0$ impacto da saúde bucal em pacientes HIV ${ }_{+}$não tem sido suficientemente documentado e Broder et al. ${ }^{5}$ apontam, dentre as dificuldades, o pouco interesse na investigação das barreiras existentes ao desenvolvimento de programas de saúde bucal ou ao nível de cooperação e interesse na população com diagnóstico positivo para o HIV.

Broder et al. ${ }^{6}$ salientam a importância de voltar os olhares para o pacienteinfantil eadolescente e afirmam que os responsáveis pelas crianças e adolescentes têm a expectativa departicipação em programas odontológicos. Porém, uma vez iniciadas as atividades, ocorregrande evasão, principalmente pelo fato destes indivíduos terem a expectativa de tratamento odontológico curativo enão estarem preparados para considerar orientação e prevenção como uma estratégia importante.

A este fato, Phelan 7 inclui as dificuldades familiares frente à doença, uma vez que o diagnóstico positivo acarretará necessidades não apenas físicas, mas emocionais e sociais, que requerem uma interação de uma equipe multidisciplinar. N este processo, as sessões informativas e educativas devem ser elaboradas a partir dos anseiose da realidade dos indivíduo ${ }^{8-11}$, sendo importante a disponibilidade de instrumentos que reflitam adequadamente as atitudes relativas às dimensões odontológicas.

Para tanto, Balbo ${ }^{12}$ propôs uma escala odontológica a ser utilizada para avaliar a percepção, os cuidados e a promoção de saúde baseada em informações dadas pel os responsáveis por crianças e adolescentes $\mathrm{HIV}_{+}$eeste estudo se propõea avaliar a reprodutibilidadedesta escala, bem como fazer um diagnóstico do serviço e saúde bucal para os pacientes que possuem cadastro junto ao GASPA (Grupo deA poio eSolidariedade aos Portadores do vírus HIV de Araraquara).

\section{Material e métodos}

Previamenteà execução deste estudo, a entidade que presta atendimento aos portadores de HIV (GASPA) foi consultada e deu autorização, por escrito, para levantamento de dados junto às famílias cadastradas.

0 delineamento amostral adotado foi o não probabilístico. A amostra esteve constituída pela total idade de crianças e adolescentes $\mathrm{HIV}_{+}$, com registro disponível no GASPA, e cujas mães ou responsáveis concordaram em participar do estudo por meio da aceitação do termo de consentimento livre e esclarecido, totalizando, ao final do estudo, 27 participantes.

0 instrumento de medida utilizado foi 0 modelo de "escala odontológica" proposta por Balbo ${ }^{12}$ e aplicada para crianças $\mathrm{HIV}_{+}$atendidas naFMRP-USP.

Esta escala se propõe a avaliar aspectos ligados à promoção de saúde, percepção, conhecimento, hábitos e cuidados que as mães/responsáveis têm em relação à saúde bucal de crianças e adolescentes soropositivas, basean do-se em questões fechadas precodificadas.

o questionário refere-se a três domínios que avaliam percepção (grau de importância dada à saúde bucal), cuidados (cuidados com a higiene bucal) e promoção (fatores relacionados à promoção de saúde bucal), calculados por um algoritmo constituído a partir da seleção de vinteitens do questionário, conforme proposto por Balbo ${ }^{12}$.

A aplicação do questionário foi realizada por um único pesquisador, previamentetreinado, em duas ocasiões, com intervalo de sete dias entre elas.

Para o cálculo da reprodutibilidade, empregou-se a estatística Kappa ${ }^{13}$.

A estatística Kappa foi estimada por ponto (k), sendo classificada segundo padrões de Landis e Koch ${ }^{14}$.

Para análise dos dados, utilizou-se o software Stata 8.0. Os resultados estão apresentados em tabelas. 
Este estudo foi aprovado pelo ComitêdeÉtica em Pesquisa da Faculdade de O dontologia de Araraquara - UNESP.

\section{Resultados}

A amostra foi composta por 27 crianças eadolescentes $\mathrm{HIV}_{+}$, sendo a maioria do sexo feminino $(62,9 \%)$. A idade média das crianças foi de 7,3 \pm 4,8 anos, com idade mínima de um e máxima de quinze anos, sendo que doze crianças apresentaram idade maior ou igual a dez anos (44,4\%).

Segundo respostas dos responsáveis por crianças e adolescentes entrevistados às questões da escala odontológica (EO), elaborou-se a Tabela 1.

Todos os respondentes afirmaram que consideram a saúde bucal importante. Porém, nota-se que, apesar disto, a maioria não procura um dentista com frequência $(70,4 \%)$, e para $25,9 \%$ das crianças, a escovação dentária não é diária. Apenas $18,5 \%$ das crianças têm seus dentes escovados por um adulto eo mesmo número utiliza fio dental. Quanto à orientação de saúde, nota-seque a maioria dos responsáveis não recebeu atendimento educativo preventivo em saúde bucal, nem informação sobre as manifestações bucais decorrentes da doença e da necessidade de melhores cuidados higiênicos devido à grande quantidade de açúcar contida nos medicamentos. Todos os responsáveis afirmaram não ter recebido informações sobre a capacidade dos medicamentos causarem xerostomia.

$\mathrm{Na}$ Tabela 2, apresenta-se o estudo da reprodutibilidade de cada item componente do questionário proposto por Balbo ${ }^{12}$.

Nas questões relativas à mãe, houve concordância máxima nos itens referentes à importância da saúde bucal, hábito de escovação diária e a presença de escova dental individual para cada membro da família. A procura de atendimento odontológico motivado por estética e para manutenção de hálito puro apresentou concordância boa.

Dentre as questões relativas à criança, observou-se concordância regular para os itens referentes à utilização do fio dental e sua frequência. As demais questões apresentaram concordância classificada como boa e ótima.

Quanto às informações relacionadas com a organização do sistema de saúde, chama atenção a dificuldade dos respondentes em relatar 0 local e o profissional que realizou orientações

Tabela 1. Distribuição de frequência $(n)$ das respostas dos responsáveis entrevistados às questões componentes da escala odontológica (EO). Araraquara (SP), 2006.

\begin{tabular}{|c|c|c|}
\hline Questões & Sim & Não \\
\hline EO1 = Você considera a saúde bucal importante? & 27 & . \\
\hline EO2 = Acha importante procurar 0 atendimento odontológico por "estética"? & 9 & 18 \\
\hline E03 = Acha importante procurar o atendimento odontológico por "hálito puro"? & 9 & 18 \\
\hline EO4 = Acha importante procurar 0 atendimento odontológico por "saúde"? & 19 & 8 \\
\hline EO5 = Acha importante procurar o atendimento odontológico por "higiene"? & 12 & 15 \\
\hline E06 = Acha importante procurar o atendimento odontológico por "evitar dor"? & 9 & 18 \\
\hline E07 = Acha importante procurar o atendimento odontológico por "evitar gastos"? & 9 & 18 \\
\hline ortante cuidar da saúde bucal de seu filho? & 27 & - \\
\hline cura o dentista com frequência? & 8 & 19 \\
\hline E010 = Vocế tem o hábito de escovar o dente diariamente? & 26 & \\
\hline EO 12 = A criança escova ou tem seus dentes esc & 21 & 6 \\
\hline E013 = A escovação dos dentes da criança é d & 20 & 7 \\
\hline E014 = Você é quem escova os dentes da criança? & 5 & 21 \\
\hline escova seus dentes? & 16 & 10 \\
\hline EO16 = 0 fio dental é usado pela criança? & 5 & 22 \\
\hline $\begin{array}{l}\text { E017 = Nos serviços de saúde, você já recebeu algum tipo de orientação e/ou atendimento } \\
\text { educativo preventivo sobre a saúde bucal de seu filho(a)? }\end{array}$ & 9 & 18 \\
\hline festações bucais decorrentes da doença? & 9 & 18 \\
\hline $\begin{array}{l}\text { E019 = Algum profissional já orientou sobre o açúcar contido nos medicamentos, ou seja, da } \\
\text { necessidade de melhores cuidados higiênicos? }\end{array}$ & 1 & 26 \\
\hline $\begin{array}{l}\text { EO20 = Algum profissional já orientou sobre a diminuição de saliva (xerostomia), ocasionada } \\
\text { pelos medicamentos? }\end{array}$ & - & 27 \\
\hline E021 = A criança já foi levada ao dentista? & 17 & 10 \\
\hline
\end{tabular}


Tabela 2. Estatística Kappa ( $\kappa$ ) aplicada a todas as questões componentes do questionário proposto por Balbo ${ }^{12}$. Araraquara (SP), 2006.

\begin{tabular}{|c|c|c|}
\hline Questões & $\mathrm{k}\left(\mathrm{IC}_{95 \%)}\right.$ & Classificação \\
\hline \multicolumn{3}{|l|}{1 - Questões relativas à mãe } \\
\hline 1.1- Você considera a saúde bucal importante? & 1,00 & Ótima \\
\hline \multicolumn{3}{|l|}{1.2 - Você acha importante procurar atendimento odontológico por: } \\
\hline A - Estética & 0,65 & Boa \\
\hline B - Saúde & 0,54 & Regular \\
\hline C - Evitar dor & 0,60 & Regular \\
\hline D - Hálito puro & 0,67 & Boa \\
\hline E - Higiene & 0,56 & Regular \\
\hline F - Evitar gastos & 0,54 & Regular \\
\hline 1.3 - Você acha importante cuidar da saúde bucal do seu filho? & 1,00 & Ótima \\
\hline 1.4 - Você procura o dentista com frequência? & 0.47 & Regular \\
\hline 1.5 - Você tem hábito de escovar os dentes diariamente? & 1,00 & Ótima \\
\hline 1.6 - Na sua casa, cada membro da família tem sua própria escova de dente? & 1,00 & Ótima \\
\hline \multicolumn{3}{|l|}{2 - Questões relativas à criança } \\
\hline 2.1 - A criança escova ou tem seus dentes escovados? & 0,76 & Boa \\
\hline 2.1.1 - Se sim, a escovação é diária? & 0,90 & Ótima \\
\hline 2.1.2 - Quem escova os dentes da criança? & 0,79 & Boa \\
\hline 2.1.3 - Quantas vezes por dia a criança escova ou tem os seus dentes escovados? & 0,67 & Boa \\
\hline 2.2 - 0 fio dental é utilizado pela criança? & 0,60 & Regular \\
\hline 2.2.1 - Se sim, qual a frequência que o fio dental é utilizado pela criança? & 0,46 & Regular \\
\hline 2.3 - A criança faz uso de mamadeira noturna? & 0,92 & Ótima \\
\hline 2.3.1 - Se sim, com que frequência? & 0,86 & Ótima \\
\hline 2.3.2 - Qual é a composição da mamadeira? & 1,00 & Ótima \\
\hline \multicolumn{3}{|l|}{2.4 - 0 que a criança come na mai or parte das refeições? } \\
\hline A - arroz & 0,78 & Boa \\
\hline B - feijão & 0,78 & Boa \\
\hline C - carne & 0,79 & Boa \\
\hline$D-$ ovos & 0,57 & Regular \\
\hline E - verduras & 0,64 & Boa \\
\hline $\mathrm{F}$ - legumes & 0,74 & Boa \\
\hline G - leite & 1,00 & Ótima \\
\hline $\mathrm{H}$ - frutas & 0,71 & Boa \\
\hline I - outros & 0,65 & Boa \\
\hline 2.5 - A criança come doces ou alimentos que contenham açúcar? & 0,65 & Boa \\
\hline $\begin{array}{l}\text { 2.5.1 - Se sim ou raramente, qual a frequência com que a criança come doces ou } \\
\text { alimentos que contenham açúcar? }\end{array}$ & 0,63 & Boa \\
\hline \multicolumn{3}{|l|}{3 - Informações relativas à organização do sistema de saúde } \\
\hline $\begin{array}{l}3.1 \text { - Você já recebeu algum tipo de orientação e/ou atendimento educativo preventivo } \\
\text { sobre a saúde bucal do seu filho? }\end{array}$ & 0,65 & Boa \\
\hline 3.1.1 - Se sim, onde? & 0,54 & Regular \\
\hline 3.1.2 - Que tipo de orientação? & 0,73 & Boa \\
\hline 3.2 - Algum profissional já orientou sobre as manifestações bucais decorrentes da doença? & 0,52 & Regular \\
\hline 3.2.1 - Se sim, qual foi o profissional? & 0,39 & Sofrível \\
\hline $\begin{array}{l}3.3 \text { - Algum profissional já orientou sobre o açúcar contido nos medicamentos, ou seja, da } \\
\text { necessidade de melhores cuidados higiênicos? }\end{array}$ & 0,29 & Sofrível \\
\hline 3.3.1 - Se sim, qual foi o profissional? & 0,13 & Fraca \\
\hline $\begin{array}{l}3.4 \text { - Algum profissional já orientou sobre a diminuição da saliva (xerostomia), ocasionada } \\
\text { pelos medicamentos? }\end{array}$ & 1,00 & Ótima \\
\hline 3.4.1 - Se sim, qual foi o profissional? & 1,00 & Ótima \\
\hline
\end{tabular}




\begin{tabular}{|c|c|c|}
\hline Questões & $\mathrm{k}\left(\mathrm{IC}_{95 \%)}\right.$ & Classificação \\
\hline 3.5 - A criança já foi levada ao dentista? & 1,00 & Ótima \\
\hline 3.5.1 - Se não, porquê? & 0,92 & Ótima \\
\hline 3.5.2 - Se sim, qual o dentista? & 0,70 & Boa \\
\hline 3.5.3 - O dentista foi informado sobre a doença? & 1,00 & Ótima \\
\hline 3.5.4 - Se sim, como o profissional reagiu? & 0,82 & Ótima \\
\hline 3.6 - Teve dificuldade em conseguir o tratamento odontológico? & 1,00 & Ótima \\
\hline 3.7 - Você acha que o atendimento foi diferente? & 1,00 & Ótima \\
\hline 3.7.1 - Se sim, porquê? & 1,00 & Ótima \\
\hline 3.7.2 - Isto criou algum tipo de constrangimento? & 1,00 & Ótima \\
\hline $\begin{array}{l}3.8 \text { - Alguma vez sentiu que seu filho(a) foi rejeitado(a) ou desrespeitado(a) no } \\
\text { tratamento odontológico? }\end{array}$ & 1,00 & Ótima \\
\hline 3.9 - Alguma vez teve o tratamento dentário do seu filho negado? & 0,93 & Ótima \\
\hline 3.9.1 - Se sim, qual foi o motivo alegado? & 1,00 & Ótima \\
\hline
\end{tabular}

educativas preventivas, bem como a necessidade deatenção à higiene bucal devido ao conteúdo de açúcar presente nos medicamentos. A concordância das demais questões foi considerada boa e ótima.

A estatística Kappa (k) aplicada aos domínios componentes da escala odontológica (EO) está exposta na Tabela 3.

Verificou-se boa concordância entre as respostas apenas para o domínio referente à promoção de saúde.

\section{Discussão}

0 delineamento amostral utilizado, não probabilístico, poderia representar, numa primeira análise, uma limitação do estudo quanto a sua validade externa. Entretanto, baseado no fato do total de crianças e adolescentes $\mathrm{HIV}_{+}$configurar uma amostra de pequeno porte, a opção do pesquisador foi trabalhar com todas elas, desde que houvesse o consentimento por parte dos responsáveis. Por outro lado, os autores proponentes do questionário ${ }^{12}$ se utilizaram de amostragem por conveniência e Balbo et al. ${ }^{15}$ também apresentam dados coletados a partir deste mesmo tipo de amostra; portanto, optou-se pela realização do estudo.

O tamanho da amostra utilizada representa 0 total de crianças e adolescentes $\mathrm{HIV}_{+}$, cadastrados junto ao GASPA do município de Araraquara (SP), cujos responsáveis concordaram em participar. Entretanto, o número de participantes é insuficiente para a realização da estimativa
Tabela 3. Estatística Kappa ( $\kappa$ ) aplicada aos domínios componentes da escala odontológica (EO) proposta por Balbo ${ }^{12}$. Araraquara (SP), 2006.

\begin{tabular}{lcc}
\hline Domínios - Escala odontológica & $\boldsymbol{\kappa}$ & Classificação \\
\hline Percepção & 0,48 & Regular \\
Cuidado & 0,21 & Sofrível \\
Promoção & 0,64 & Boa \\
\hline
\end{tabular}

do valor Kappa por intervalo de confiança. Por essa razão, a confiabilidade do instrumento proposto por Balbo ${ }^{12}$, para implementação do mesmo por parte dos serviços de saúde, foi verificada pela estatística Kappa, por ponto.

$\mathrm{Na}$ amostra estudada, 100,0\% dos respondentes afirmaram considerar a saúde bucal importante, bem como cuidar da saúde bucal de seu filho (Tabela 1). Entretanto, quando questionouse o motivo considerado importante para procura de atendimento odontológico, pode-se notar que não houve uma opinião de consenso ea concordância entre as respostas dadas nos distintos momentos foi classificada como boa apenas para os itens "Estética"e "Hálito puro", enquanto os demais apresentaram reprodutibilidade regular, o que pode ser reflexo da falta de informação sobre hábitos de saúde bucal (Tabela 2).

Chama atenção queoito indivíduos (29,6\%) afirmaram não procurar o dentista com frequência, o que pode ter ocorrido por desinformação, 
por inviabilidade econômica ou devido às diversidades de problemas ocasionados em todos os aspectos da vida pelo impacto da SI DA. Deacordo com $\mathrm{H}$ astreiter e Jiang ${ }^{16}$, a dificuldade financeira tem sido um limitador do acesso a serviços

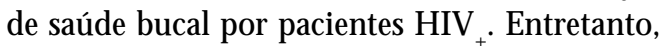
os autores apresentam evidências de que a falta de visitas regulares aos serviços de saúde bucal pode ser mais dispendiosa do que o tratamento regular, alertando os pacientes e os serviços de saúde para este fato.

Com relação aos cuidados de higiene bucal decrianças, a literatura ${ }^{15,17}$ tem recomendado que crianças menores de dez anos tenham seus dentes escovados ou sua escovação monitorada por um adulto, uma vez que as mesmas ainda não possuem habilidade manual nem maturidade suficiente para garantir a execução de uma escovação adequada. N este estudo, apenas em 18,5\% dos casos foi relatada a presença do responsável durante a escovação (Tabela 1).

Outro aspecto a ser considerado éa não utilização do fio dental pela maioria das crianças ( Tabela 1), concordando com os achados de Bal bo et al. ${ }^{15}$. Na Tabela 2, observou-se uma concordância regular para os itens referentes à utilização do fio dental esua frequência, o que pode ser reflexo da dificuldade dos cuidadores em assumir a não utilização do mesmo pela criança. A dificuldade de crianças e de adolescentes em se habituar à utilização do fio dental têm sido apontadana literatura; entretanto, tratando-se de crianças $\mathrm{HIV}_{+^{\prime}}$ esta situação requer maior atenção, uma vez que a presença de placa bacteriana, aliada à debilidade imunológica, pode corroborar para o desenvolvimento de infecções oportunistaš ${ }^{2,18,19}$.

Quanto à falta de informações sobre atendimento educativo preventivo, manifestações bucais decorrentes da doença, efeitos na cavidade bucal pelo consumo de medicamentos (questões EO 17, EO18, EO 19 eEO20) (Tabela 1), pode-seatribuir o fato à não existência de um profissional responsável pelasaúdebucal das crianças HIV $\mathrm{V}_{+}$junto à equipe multidisciplinar de saúde. Ribeiro ${ }^{20}$ e Zabos e Trinh ${ }^{21}$ ressaltam a necessidade de elaboração de programas de promoção de saúde, pautados na educação e motivação e voltados para 0 atendimento das necessidades de pacientes $\mathrm{HIV}_{+}$.

De acordo com Jandinski et al. ${ }^{22}$, M adigan et al. ${ }^{23}$, Castro et al. ${ }^{24}$ e Ribeiro et al. ${ }^{2}$, a experiência de cárie e gengivite em crianças infectadas pelo HIV é elevada e esta falta de informação pode levar ao agravamento desta situação, pautada no fato de que estas crianças e adolescentes fazem um al to consumo de medicamentos contendo açúcar, resultando na diminuição do fluxo salivar, causada pelos mesmos e por hábitos inadequados de higiene bucal 25 .

Quanto às informações relacionadas com a organização do sistema de saúde, chama atenção a dificuldade dos respondentes em relatar 0 local e o profissional que realizou orientações educativas preventivas e quanto ao conteúdo de açúcar nos medicamentos, o que pode denotar falta de programas contínuos de orientação voltados para o paciente pediátrico $\mathrm{HIV}_{+}$.

A pesar das estimativas da consistência interna da escala odontológica realizadas por Balbo ${ }^{12}$ e Balbo et al. ${ }^{15}$ terem conferido ao instrumento bons resultados, estas não garantem à escala total qualidade metodológica. Assim, para a certificação da confiabilidade do referido instrumento, deve-se proceder, além destas estimativas, 0 estudo da reprodutibilidade de cada domínio componente da escala odontológica (EO).

A concordância apresentada para cada um dos domínios percepção, cuidado e promoção foi de $0,48,0,21$ e 0,64 , respectivamente, 0 que aponta para uma reprodutibilidade abaixo do desejável para os domínios percepção e cuidado.

Destemodo, a par da constatação da necessidade de inclusão, nos programas de saúde de

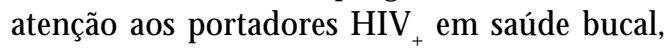
sugere-sequemais estudos sejam realizados para a investigação da reprodutibilidade da escala odontológica, a fim de que o algoritmo proposto para cada domínio possa ser avaliado e, se necessário, reformulado até que 0 instrumento atinja a confiabilidade necessária para o levantamento das informações da percepção, dos cuidados e de promoção de saúde bucal por parte de cuidadores de crianças e adolescentes $\mathrm{HIV}_{+}$.

\section{Conclusão}

Frente aos achados, sugere-se a necessidade de que a saúde bucal de portadores HIV + seja integrante da atenção a eles destinada. A reestruturação das questões componentes dos algoritmos propostos pela escala odontológica para as dimensões de percepção e cuidado devem ser reavaliadas, após a inclusão da saúde bucal em programas de atendimento de portadores $\mathrm{HIV}_{+}$. 


\section{Colaboradores}

JADB Campos e LCM Loffredo participaram igualmente de todas as etapas da elaboração do artigo.

\section{Referências}

1. Rachid M, Schechter M. M anual de HIV/AIDS. Rio de Janeiro: Revinter; 1998.

2. Ribeiro AA, Portela M, Souza IP. Relação entre biofilme, atividade de cárie e gengivite em crianças HIV +. Pesqui. O dontol. Bras. 2002; 16(2):144-150.

3. Chapple ILC, Hamburger J. The significance of oral health in HIV disease. Sex Transm Infect 2000; 76(4):236-243.

4. Coulter ID, Heslin KC, Marcus M, Hays RD, Freed J, Der-M artirosian C, Guzmán-Becerra N, Cunningham WE, Andersen RM, Shapiro M F. Associations of self-reported oral health with physical and mental health in nationally representative sample of HIV persons receiving medical care. Qual Life Res 2002; 11(1):57-70.

5. Broder $\mathrm{HL}$, Catalanotto $\mathrm{FA}$, Reisine $\mathrm{S}$, Variagiannis E. Compliance is poor among HIV-infected children with unmet dental needs. Pediatr Dent 1996; 18(2):137-138.

6. Broder HL, Russell SL, Varagiannis E, Reisine ST. Oral health perceptions and adherence with dental treatment referrals among caregivers of children with HIV. AIDS Educ Prev 1999; 11(6):541-551.

7. Phelan JP. Oral health care for adults, adolescents and children with HIV infection. New York: Aids Institute/D epartment of Health; 1998.

8. Souza L. A saúde e doença no dia-a-dia do povo. Caderno do CEAS 1982; 77:18-23.

9. Portillo JAC, Paes AM C. Autopercepção de qualidade de vida relativa à saúde bucal. Rev Bras Odontol Saúde Coletiva 2000; 1(1):75-88.

10. Bensing JM, Visser A, Saan H. Patient education in the Netherlands. Patient Educ Couns 2001; 44(1):15-22.

11. Myburgh NG, Hobdell MH, Laloo R. African countries propose a regional oral health strategy: The Dakar Report from 1998. Oral Dis. 2004; 10(3):129-137.

12. Balbo PL. Epidemiologia de fatores sociais relacionados à saúde bucal relatados pelas mães ou pelos responsáveis por crianças HIV +/AIDS atendidas no HCRP [tese]. Ribeirão Preto (SP): Faculdade de M edicina de Ribeirão Preto, USP; 2006.

13. Light RJ. M easures of response agreement for qualitative data: some generalizations and alternatives. Psychol Bull 1971; 76:365-377.

14. Landis JR, Koch GG. The measurement of observer agreement for categorical data. Biometrics 1977; 33:159-174.
15. Balbo PL, Rodrigues-Júnior AL, Cervi MC. Caracterização dos cuidadores de crianças HIV /AIDS abordando a qualidade de vida, a classificação socioeconômica e temas relacionados à saúde bucal. Cien Saude Colet 2007; 12(5):1301-1307.

16. Hastreiter RJ, Jiang P. Do regular dental visits affect the oral health care provided to people with HIV? J Am Dent Assoc 2002; 133:1343-1350.

17. Ottley C. Improving children's dental health. J Fam H ealth Care 2002; 12(5):122-125.

18. Eldridge K, Gallagher JE. Dental caries prevalence and dental health behavior in HIV infected children. Int. J. Paediat. Dent. 2000; 10:19-26.

19. Shetty K. Recommendations for the dental management of HIV-infected children and adolescents. HIV Clinician 2004; 16(2):1-7.

20. Ribeiro AA. Avaliação de um programa de promoção

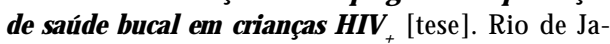
neiro (RJ): Faculdade de Odontologia, Universidade Federal do Rio de Janeiro; 2000.

21. Zabos GP, Trinh C. Bringing the mountains to M ohammed: a mobile dental team serves a community-based program for people with HIV/AIDS. Am J Public Health 2001; 91(8):1187-1189.

22. Jadinski J, Catalanotto F, Murray P, Katz R, Varagiannis E. Oral pathology in pediatrics AIDS. [abstract 2705] J Dent Res 1994, 73:440.

23. Madigan A, Murray PA, Houpt M, Catalanotto F, Feuerman $M$. Caries experience and cariogenic markers in HIV-positive children and their siblings. Pediatr Dent 1996; 18(2):129-136.

24. Castro GF, Souza IP, Chianca TK, Hugo R. Avaliação de um programa de prevenção de cárie em

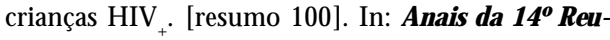
nião Anual da Sociedade Brasileira de Pesquisa O dontológica; 1997; São Paulo.

25. Maguire A, Rugg-Gunn AJ, Butler TJ. Dental health of children taking antimicrobial and non-microbial liquid oral medication long-term. Caries Res 1996; 30:16-21.

Artigo apresentado em 26/12/2007

Aprovado em 09/07/2008

Versão final apresentada em 30/10/2008 